\title{
Agent cognition through micro-simulations: Adaptive and tunable intelligence with NetLogo LevelSpace
}

\author{
Bryan Head ${ }^{1}$ and Uri Wilensky ${ }^{1,2}$ \\ 1 Center for Connected Learning and Computer-Based Modeling, Northwestern \\ Institute of Complex Systems, Department of EECS, Northwestern University, \\ Evanston IL, USA \\ Homepage: https://ccl.northwestern.edu/ \\ bryan.head@u.northwestern.edu, uri@northwestern.edu \\ 2 Department of Learning Sciences, Northwestern University, Evanston IL, USA
}

\begin{abstract}
We present a method of endowing agents in an agent-based model (ABM) with sophisticated cognitive capabilities and a naturally tunable level of intelligence. Often, ABMs use random behavior or greedy algorithms for maximizing objectives (such as a predator always chasing after the closest prey). However, random behavior is too simplistic in many circumstances and greedy algorithms, as well as classic AI planning techniques, can be brittle in the context of the unpredictable and emergent situations in which agents may find themselves. Our method, called agent-centric Monte Carlo cognition (ACMCC), centers around using a separate agent-based model to represent the agents' cognition. This model is then used by the agents in the primary model to predict the outcomes of their actions, and thus guide their behavior. To that end, we have implemented our method in the NetLogo agent-based modeling platform, using the recently released LevelSpace extension, which we developed to allow NetLogo models to interact with other NetLogo models. As an illustrative example, we extend the Wolf Sheep Predation model (included with NetLogo) by using ACMCC to guide animal behavior, and analyze the impact on agent performance and model dynamics. We find that ACMCC provides a reliable and understandable method of controlling agent intelligence, and has a large impact on agent performance and model dynamics even at low settings.
\end{abstract}

Keywords: agent-based modeling, artificial intelligence, agent cognition, multi-level agent-based modeling, NetLogo

\section{Introduction}

Agent-based modeling (ABM) has long proven to be a powerful method for simulating complex systems [3, 8, 15. Over the last decade, multi-level agent-based modeling (MLABM) has extended this power by enabling researchers to create systems of connected ABMs [9]. This allows one to model a system with multiple 
components or levels by creating separate ABMs for each component that are then connected. We recently released LevelSpace [5, which brings the ability to integrate many different ABMs to NetLogo [14, one of the most widely used ABM platforms. Here, we demonstrate how to leverage the power of MLABM in order to define sophisticated and tunable cognitive systems for guiding agent behavior.

Agent cognition differs from classic artificial intelligence algorithms in that the goal is not to generate the most optimal course of action. Instead, it is desirable to have a level of intelligence that is appropriate for the agents in question. For instance, agents representing humans should have significantly different cognitive capabilities than agents representing sheep, which should, in turn, have significantly different capabilities than agents representing ants. Furthermore, the task is complicated by the fact that, due to the subject matter of most ABMs, agents exist in complex environments with many other agents, such that the patterns of their world are an emergent result of the collective actions of those agents. Making matters more difficult, agents are typically only aware of local information. Thus, agents will find themselves in surprising and unexpected circumstances, and must continue to act reasonably in those circumstances. Hence, our goal is to design a method of agent cognition in which:

1. Agents are given goals and information about their local state, and they determine what actions to take.

2. Agents have a tunable level of "intelligence".

3. What agents know about the world and how they think the world works is definable. In other words, agents should often have simplified understandings of the world.

4. Agents behave reasonably when in unexpected circumstances, or when something surprising occurs.

5. Ideally, the agents' cognitive processes should be inspectable and understandable. Researchers should be able to understand why agents are doing what they are doing.

Our method, agent-centric Monte Carlo cognition (ACMCC), accomplishes these goals by defining an ABM that represents how an agent thinks the world works. By leveraging MLABM, agents then use this cognitive ABM to make short term predictions about what will happen when they choose different actions. The agents will then make their final action decision based on the predicted outcomes of each action.

\section{Related Work}

Under one perspective, an ABM defines a Markov chain, a small part of which is observable by each agent. Hence, the framework of partially observable markov decision processes (POMDPs) may be applicable in this context [7]. Indeed, MDPs have been used to model decision making of agents in dynamic environments in other works. For instance, [1] combines POMDPs with reinforcement 
learning to perform dynamic decision making for a single agent in a stochastic environment. Further, [6] extends MDPs to work with multiple agents via Markov games. MDPs are often solved by computing the sum of discounted future reward values at each state (via dynamic programming). However, such strategies are infeasible in the context of ABMs as, typically, even the local area which an agent can observe has a vast state-space, due to the number of agents and the fact that agents are often defined by continuous attributes, and the rate at which new information is encountered. Furthermore, even if it were possible to find the optimal solution to the POMDPs involved, it would not be desirable to do so, as agents typically have bounded rationality by design. That said, our work may be characterized as using Monte Carlo simulations to approximate the solutions to POMDPs of each agents' surroundings.

Monte Carlo tree search (MCTS) has proven to be a particularly powerful method to address decision processes, especially in the context of games [2]. Notably, MCTS was combined with deep reinforcement learning techniques to create the first super-human Go playing program [11, 12. Fundamentally, MCTS works by using Monte Carlo "playouts" to determine the expected value of each possible move the player can take. It then uses those expected values to guide future playouts, so that they focus on more promising paths. The technique may be further augmented with other methods (such as deep neural networks estimating the values of states) to further guide the playouts. In this work, we take a similar Monte Carlo approach to sampling the eventual value of actions, but use a pure Monte Carlo strategy instead. Section 7 discusses extending this work to incorporate advancements in MCTS.

44 also demonstrated a method of defining agent cognition via MLABM using an early version of LevelSpace. In that work, agents sent information about their surroundings to simple neural network models. The output of the neural networks would then determine what action the agents would take. This work has several advantages compared to that, though they are complimentary methods, as discussed in Section 7. First, this method requires no training. Second, this method uses explicit cognitive representations. That is, the objects of the agents' cognition are of the same form as the objects of the world (entities in an ABM). When a sheep is considering the actions of a wolf, a wolf agent exists in their cognitive model. Furthermore, we can directly observe why an agent does what it does, and what it expects to happen for different actions. This is in sharp contrast with a neural network, in which the agent's knowledge is implicitly embedded in the weights of the neurons. Finally, this method offers a parameter that directly corresponds with the cognitive capabilities of the agents: namely, the number of simulations the agent runs. In contrast, the number and size of the layers in a neural network controls the cognitive capabilities of the agents, but how that translates into concrete differences in cognition and behavior is opaque. 


\section{Method}

ACMCC works as follows:

1. The modeler defines what actions an agent can take (e.g., turn left, turn right, go forward, eat, etc.), what the agent knows/believes (e.g., what the agent can see), what the agent is trying to do (e.g., maximize food intake while staying alive), and how the agent thinks the world works via a cognitive model defined by a separate agent-based model. That is, this cognitive model may be a simplified version of the main ABM, which operates according to mechanisms as understood by the agent. Thus, the agent should be able to use this cognitive model to make predictions about what will happen in the full model.

2. During each tick of the simulation, each agent runs a settable number of rollouts, or short simulations of their surroundings, using its cognitive model, with initial conditions based on their surroundings. During these rollouts, the agent selects random actions and tracks how well they meet their objectives as a consequence of those actions. The agent's performance is evaluated based on reward: during a rollout, the agent will receive a reward based on what happens (the modeler defines the reward based on the agent's objectives).

3. The agent then selects an action based on the results of these rollouts.

See Figure 2 for an example of these rollouts.

A significant advantage of this method is that it gives researchers several tunable parameters that precisely control agents" "intelligence", such as the number of rollouts to run and the length of each rollout. Having such control over agents' intelligence allows modelers to, for instance, naturally adjust agents' cognitive capabilities based on what is reasonable for those agents, or have an evolvable "intelligence" parameter that directly correlates to the agents' cognitive capabilities. Note that we do not claim that this is how the actual cognitive processes of the modeled agents work. Rather, this method simply gives modelers a practical method of controlling the sophistication of agents' decision making abilities.

\section{Model}

In order to demonstrate our method, we extend the Wolf Sheep Predation model found in the NetLogo Models Library [13. Wolf Sheep Predation is a basic predator-prey model consisting of wolves, sheep, and grass, run on a two dimensional toroidal world. Wolves and sheep are point-like agents that move in continuous space, while grass exists as a binary value on a grid (either grass is present at a particular cell or it is not). In the original model, wolves eat sheep, sheep eat grass, and grass regrows at a fixed rate. When agents eat, they acquire energy, and when agents move, they lose a small amount of energy. Wolves and sheep reproduce based on a probability each tick, splitting their energy with their child. All movement is random: each tick, agents turn left or right by a random angle less than 45 and take a step forward of unit length. If an agent 


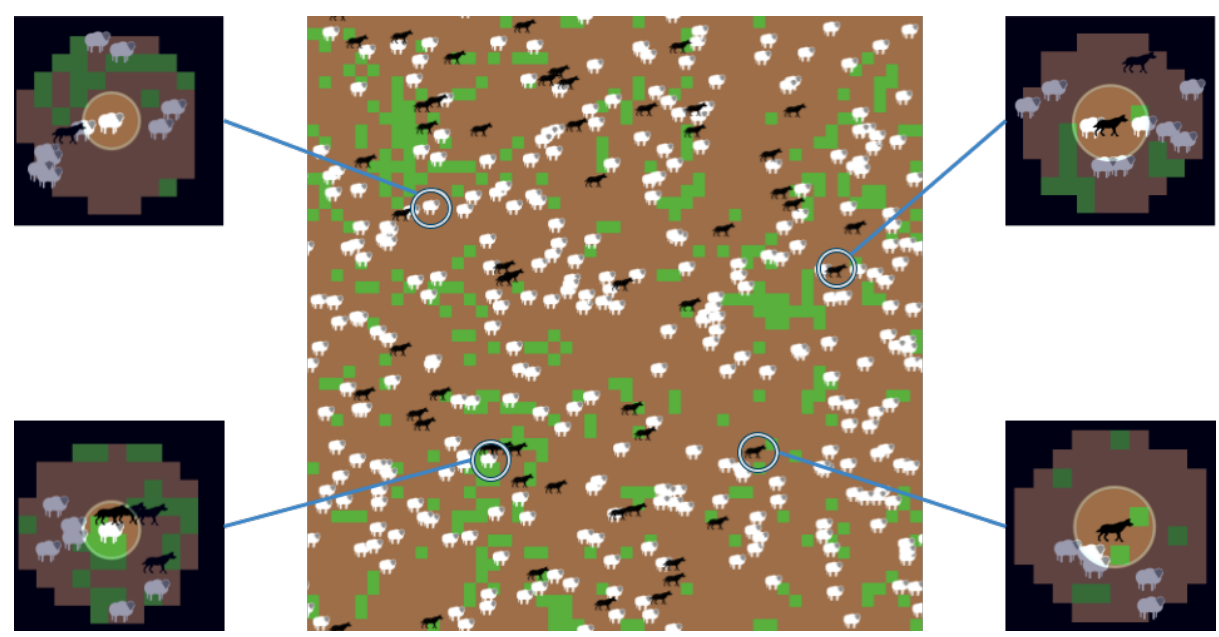

Fig. 1: A state from the modified Wolf Sheep Predation model. The initial state of the cognitive model for each of the highlighted agents is shown.

runs out of energy, it dies and is removed from the world. If an agent is eaten, it dies and is removed from the world.

In our extension of the model, the random movement is replaced with ACMCC. Agents have the following actions available to them:

1. Turn right 30, take a step forward of unit length, and eat if possible.

2. Take a step forward of unit length, and eat if possible.

3. Turn left 30, take a step forward of unit length, and eat if possible.

Reproduction still occurs based on a probability.

In order to decide what actions to take each tick, agents use a simple cognitive model. First, agents are given a vision radius; their cognitive model will be initialized with what they see in this radius each tick. The cognitive model is a simplified version of the original Wolf Sheep Predation. The world represented is much smaller than the full model: a little bit larger than their vision radius. It is initialized with the agents surroundings: the positions and headings of the surroundings wolves and sheep, as well as the positions of the live and dead grass, and finally the position, heading, and energy of the agent itself. Furthermore, this model only includes factors of which the agents would plausibly be aware of. The cognitive model thus makes the following simplifications:

- Only the energy of the primary agent (called the ego) is tracked. This is because the ego cannot observe the energy of the other agents.

- There is no reproduction. However, our model could be further extended to make reproduction an action, and have the child's well-being incorporated into the rollout reward-function. It was left out for simplicity and because that it is reasonable to think that the possibility of suddenly reproducing is not factored into the decision of which piece of grass to go for. 


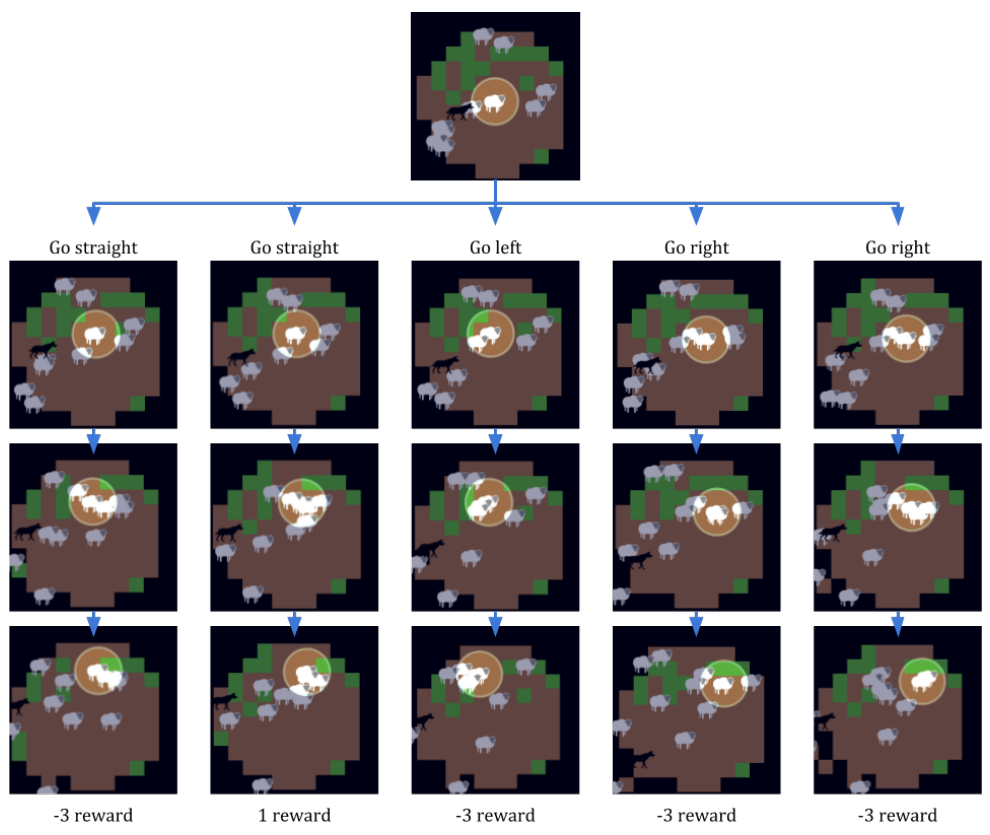

Fig. 2: The tree of rollouts performed by the highlighted sheep, which is facing north. The rollouts progress from top to bottom, with the first row showing the state after 1 tick, and the last row showing the final state. The sheep's initial actions and final reward values are shown (without discounting).

- There is no grass regrowth. Again, the ego cannot observe the regrowth state of the grass, and these are short-term simulations.

- There is nothing outside of the ego's vision radius. Should an agent move past the vision radius during a rollout, the grass at that point is filled in randomly based on the observed density of grass.

All agents in the cognitive model act randomly. While there are many interesting possibilities for selecting actions of agents in rollouts, such as further embedding cognitive models (thus giving agents a kind of theory of mind), or refining rollout decision making based on past rollouts (as in MCTS) we chose to begin with the simplest approach: random action in rollouts. Reward in the rollouts is calculated as the agents change in energy, with a discounting factor, so that energy acquired in the first tick counts more than energy acquired in the second, and so forth. Furthermore, death is given an automatic reward value of -1000 .

The main predator-prey model and cognitive model are then combined as follows. Each tick of the predator-prey model proceeds as in the original Wolf Sheep Predation, save that agents send information about their surroundings to their cognitive model, which then runs a set number of rollouts of a set length, and reports the resulting reward values. The agent then takes the action that 
had the highest mean reward. See Figure 1 for a state of the predator-prey model and the corresponding initial state of several of the cognitive models. Figure 2 shows the various rollouts performed by the cognitive model of a sheep at that tick. Through these rollouts, the sheep finds that the two sheep coming from the east almost always eat the patch of grass to the northeast, and the sheep coming from the north almost always eat the grass to the north-northwest. Thus, the only grass the sheep manages to successfully eat is the northernmost (final image in the second column). Thus, the sheep decides to go straight. In this way, ACMCC allows for agents to perform sophisticated decision making where a greedy algorithm would have failed (because the closest grass would have been eaten by other sheep first).

\section{Results}

In order to understand the effects of the cognitive model on Wolf Sheep Predation, several experiments were performed. In each experiment, one agent-type had the number and length of its rollouts varied while the other agent-type was kept random. The efficiency of the agents in various tasks as well as population levels were recorded. Two measurements of efficiency were used: one for sheep and one for wolves. Sheep efficiency was measured as the ratio of the amount of grass eaten in a tick to the amount of grass we would expect to be eaten by random sheep, given grass density and number of sheep: grass-eaten / (num-sheep * grass-density). Similarly, wolf efficiency was measured as the ratio of the amount of sheep eaten in a tick to the amount of sheep we would expect to be eaten by random wolves, given sheep density and number of wolves: sheep-eaten / (num-wolves * sheep-density). This measure was used as it controls for population dynamics: it measures how well the agents are doing compared to how well we would expect random agents to be doing. Thus, in both cases, an efficiency of 1 is random. However, as there are relatively few wolves (in the base model, wolves oscillate between 50 and 100, while sheep oscillate between 120 and 200) with relatively few prey (the sheep's prey, grass, covers around 1000 locations at any given tick), this number rests below 1 for the baseline case (at around 0.76) due to the fact that wolves and their prey are discrete entities. Sheep, on the other hand, are more numerous with an evenly spread food source, and thus their baseline rests close to the theoretical value of 1 , at 0.93 .

For both wolves and sheep, number of rollouts was varied from 1 to 20 (note that performing only a single rollout is equivalent to random behavior) and the length of rollouts was varied from 0 to 5 . Vision radius was set to 5 for both agent types. Default parameter settings (as found in the NetLogo Models Library) were used for all other parameters. Runs were carried out for 2,000 ticks. 20 repetitions were performed for each combination of parameters. Figure 3 shows the efficiency results for both agent-types.

Finally, in order to begin to understand the impact of the cognitive model on the dynamics of the model, we examine the sheep population. Using the 


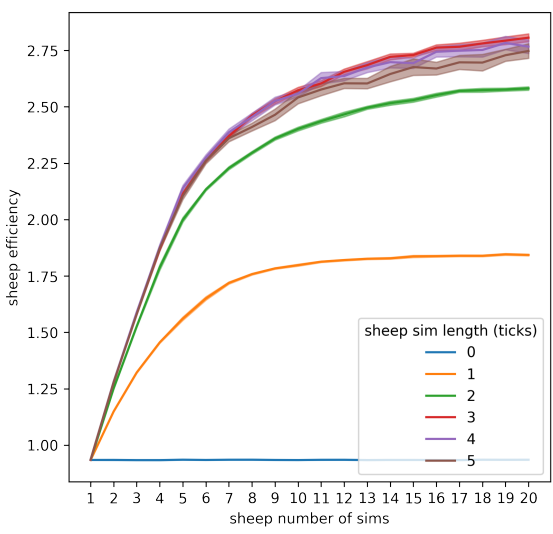

(a) Sheep using rollouts, wolves random

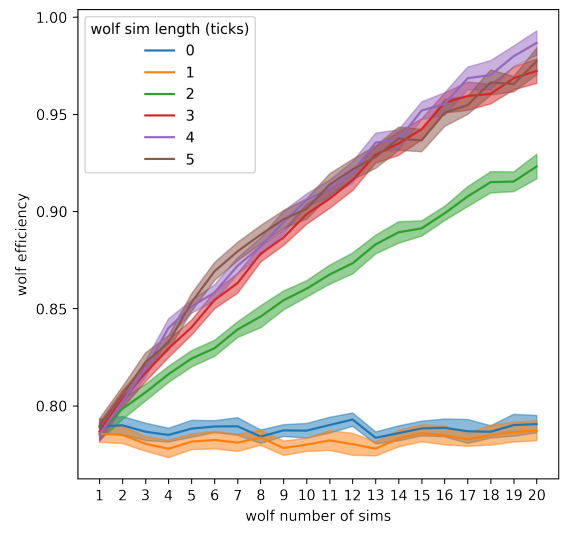

(b) Wolves using rollouts, sheep random

Fig. 3: Mean efficiency of sheep and wolves at eating their respective foods (grass and sheep) for various combinations of number and length of rollouts. Bands show $95 \%$ confidence intervals. Note that the y-axes have different scales.

same runs as above, Figure 4a shows the mean population of sheep for each combination of number and length of sheep rollouts. In contrast, Figure $4 \mathrm{~b}$ shows mean sheep population in response to wolf cognitive abilities. Means are taken from tick 500 onwards in each run, to allow for the system to reach stability.

\section{Discussion}

We find that the number of simulations has a direct and monotonic impact on agent performance, and thus works well as an "intelligence" parameter. Furthermore, even a small number of short rollouts dramatically increases performance in sheep. Thus, it is possible to give hundreds of agents fairly sophisticated short-term reasoning in this way without too high of a performance impact: all simulations performed here are runnable in real-time on a standard, modern laptop. Rollout length appeared to reach maximum efficacy at around three ticks. This is not surprising; the longer rollouts are run for, the less accurate they will be, especially as agents could only see to distance 5 . This invites the question of how to do longer term predictions about the future; this is discussed in Section 7 .

While wolves were not helped as much as sheep, their performance was still improved with a relatively small number of short rollouts. Regardless, their poorer performance is not surprising: catching a moving prey is much more difficult than catching a stationary one, or even than avoiding being caught. Nevertheless, wolf performance is impressive considering the branching factor and their poor model of sheep behavior. Methods for overcoming their natural disadvantage are discussed in Section 7 . 


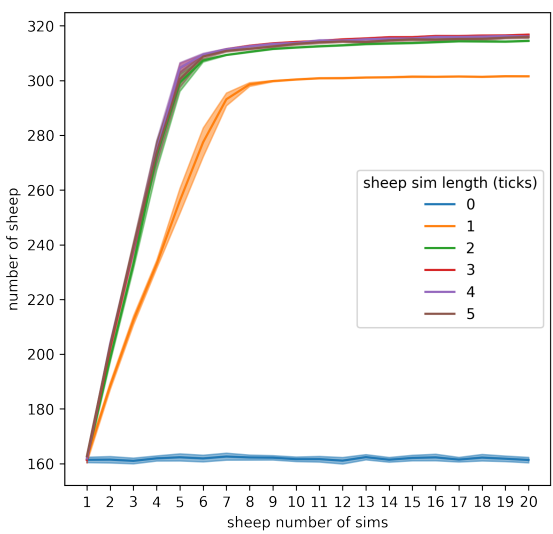

(a) Sheep using rollouts, wolves random

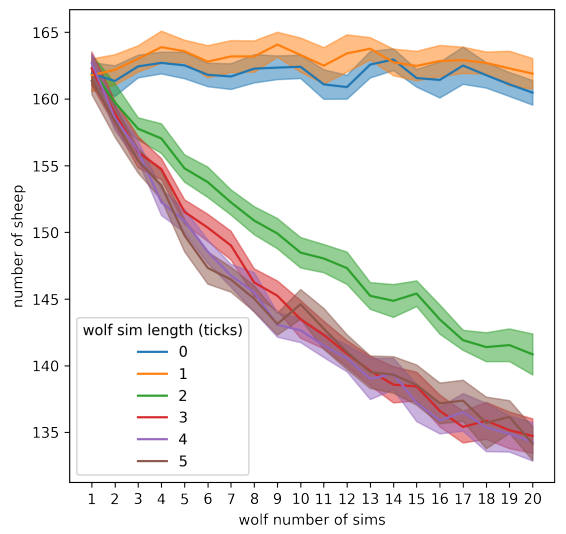

(b) Wolves using rollouts, sheep random

Fig. 4: Mean sheep population for various combinations of number and length of rollouts performed by sheep and wolves. Bands show $95 \%$ confidence intervals. Note that the y-axes have different scales.

As shown in Figure 4b, as the wolves become smarter, their food supply significantly drops. This is particularly significant as fewer sheep means fewer sheep reproducing, and thus less food for the wolves being introduced each tick. This is unlike the sheep's food source, which regrows at each location at a fixed rate, and thus, the fewer locations occupied by grass, the faster it is introduced into the system. The effect of wolf behavior on sheep population highlights the difficulties of applying cognitive systems; due to the aggregate-level feedback loop in the system, what's beneficial to the individual can be harmful to the group.

More broadly, these results indicate that this is a promising strategy for giving agents more sophisticated, yet tunable cognitive capabilities in a natural way, using nothing but agent-based modeling.

\section{$\begin{array}{ll}7 & \text { Future work }\end{array}$}

While this work lays a solid foundation for a novel approach to agent cognition, it can be extended in many interesting ways.

First, as the agents are only performing a handful of rollouts, it is important that those rollouts focus on promising/likely actions and futures. This is particularly salient in the case of the wolves, who have the more difficult task of catching a moving target. There are a number of established ways of accomplishing this. First, in MCTS, this is accomplished by using the results of past rollouts to guide future rollouts. There are some difficulties here: with the few number of rollouts and the continuous state-space of many ABMs, it is unlikely 
for the rollouts to encounter the same state twice. Regardless, this method is immediately useful for the choice of the first action, and could be modified to work on future actions by either compressing states (using a learned encoder) or scoring sequences of actions, regardless of state.

Another method that has recently proven to be highly effective is combining MCTS with neural networks, as was done in AlphaGo [11 and AlphaGo Zero 12. In ABM, neural networks could be trained to both select likely actions for the ego, as well as to predict the behavior of the other agents. This method would both improve the efficacy of rollouts, and offer a way of incorporating learning into the system, if that is desirable. Our initial work in this direction has been promising, which combines this work with the work in 4 .

Another method of better predicting the actions of other agents would be to embed another layer of ACMCC models inside the first layer. That is, agents would have a kind of theory of mind, where they try to emulate the thinking of the other agents based on what they can observe. The drawback to the naive implementation of this, however, is that it is performance intensive (initial experiments reinforce this). However, this may be circumvented by doing a kind of MCTS for each agent at the high level; that is, each agent in the cognitive model uses the results of past rollouts to improve their behavior in future rollouts, thus emulating the cognition of the other agents without performing any additional rollouts. Regardless, the naive approach of further embedding cognitive models could be quite effective for models with fewer agents that require highly sophisticated reasoning. For instance, [10 examines an approach to simulating theory of mind in a multi-agent simulation somewhat along these lines.

Another challenge is adapting this method to work with continuous action spaces. As described here, the actions must be discrete; wolves and sheep can only turn by fixed amounts, while they can turn by a continuous amount in the original model. This could be accomplished by interpolating between different discrete parameters for an action.

While the method as applied here appears to be effective for short-term reasoning, it does not perform any kind of long term reasoning. A simple way of adapting the method to perform longer term reasoning would be to decrease the accuracy of the simulation while increasing it's speed by changing its timestep. Another method would be to have the cognitive model operate on a coarser grain than the main model.

Thus, while this work lays the foundation for sophisticated agent cognition, it opens up many possible avenues of exploration as well.

\section{References}

[1] Barve, S.S.: Dynamic Decision Model using Partially Observable Markov Decision Process. International Journal of Emerging Trend in Engineering and Basic Sciences 2(1) (January 2015) $785-788$

[2] Browne, C.B., Powley, E., Whitehouse, D., Lucas, S.M., Cowling, P.I., Rohlfshagen, P., Tavener, S., Perez, D., Samothrakis, S., Colton, S.: A 
survey of monte carlo tree search methods. IEEE Transactions on Computational Intelligence and AI in games 4(1) (2012) 1-43

[3] Epstein, J.M.: Generative Social Science: Studies in Agent-Based Computational Modeling. Princeton University Press (2006)

[4] Head, B., Hjorth, A., Brady, C., Wilensky, U.: Evolving Agent Cognition with NetLogo LevelSpace. In: Proceedings of the Winter Simulation Conference. (2015)

[5] Hjorth, A., Head, B., Wilensky, U.: LevelSpace NetLogo extension. http://ccl.northwestern.edu/levelspace/index.html. Evanston, IL: Center for Connected Learning and Computer Based Modeling, Northwestern University. (2015)

[6] Littman, M.L.: Markov games as a framework for multi-agent reinforcement learning. In Cohen, W.W., Hirsh, H., eds.: Machine Learning Proceedings 1994. Morgan Kaufmann, San Francisco (CA) (1994) 157-163

[7] Lovejoy, W.S.: A survey of algorithmic methods for partially observed Markov decision processes. Annals of Operations Research 28(1) (December 1991) $47-65$

[8] Macal, C.M., North, M.J.: Agent-based modeling and simulation: ABMS examples. In: Simulation Conference, 2008. WSC 2008. Winter, IEEE (2008) $101-112$

[9] Morvan, G.: Multi-level agent-based modeling: A Literature Survey. CoRR abs/1205.0 (2013)

[10] Rabinowitz, N.C., Perbet, F., Song, H.F., Zhang, C., Eslami, S.M.A., Botvinick, M.: Machine Theory of Mind. arXiv:1802.07740 [cs] (February 2018)

[11] Silver, D., Huang, A., Maddison, C.J., Guez, A., Sifre, L., van den Driessche, G., Schrittwieser, J., Antonoglou, I., Panneershelvam, V., Lanctot, M., Dieleman, S., Grewe, D., Nham, J., Kalchbrenner, N., Sutskever, I., Lillicrap, T., Leach, M., Kavukcuoglu, K., Graepel, T., Hassabis, D.: Mastering the game of Go with deep neural networks and tree search. Nature 529(7587) (January 2016) 484-489

[12] Silver, D., Schrittwieser, J., Simonyan, K., Antonoglou, I., Huang, A., Guez, A., Hubert, T., Baker, L., Lai, M., Bolton, A., Chen, Y., Lillicrap, T., Hui, F., Sifre, L., van den Driessche, G., Graepel, T., Hassabis, D.: Mastering the game of Go without human knowledge. Nature 550(7676) (October 2017) 354-359 00000.

[13] Wilensky, U.: NetLogo Wolf Sheep Predation model. http://ccl.northwestern.edu/netlogo/models/WolfSheepPredation. Center for Connected Learning and Computer-Based Modeling, Northwestern University. Evanston, IL. (1997)

[14] Wilensky, U.: NetLogo. http://ccl.northwestern.edu/netlogo/. Center for Connected Learning and Computer-Based Modeling, Northwestern University. Evanston, IL. (1999)

[15] Wilensky, U., Rand, W.: An Introduction to Agent-Based Modeling: Modeling Natural, Social, and Engineered Complex Systems with NetLogo. MIT Press (2015) 